\title{
Del reconocimiento de la variante a la senda de lectura en el ámbito de la edición crítica digital '
}

\author{
From the Variant Realization to the Reading Path within the Realm \\ of the Digital Critical Edition
}

\author{
JAVIER MERCHÁN SÁNCHEZ-JARA \\ Universidad de Salamanca \\ España \\ javiermerchan@usal.es
}

(Recibido: 22-11-2015; aceptado: 16-03-2016)

Resumen. El presente trabajo pretende aportar una reflexión crítica en torno a la importancia de la corriente teórica conocida como New Philology, y más concretamente a las ideas expuestas en la obra In Praise of the Variant (1999), (obra fundacional de este marco teórico), como texto de capital importancia para el desarrollo de la filología en los últimos años. Pero además, queremos centrar el foco en como los aspectos y postulados teóricos que vertebran este posicionamiento metodológico, subyacen como esencia conceptual al desarrollo de los nuevos sistemas de edición crítica insertos en el paradigma digital. Así, se hace referencia a entornos digitales para la edición crítica de textos musicales, como ejemplo de sistemas que permiten incorporar las variantes presentes en los distintos testimonios, así como las particularidades materiales de los manuscritos a través de los que se transmiten, mediante herramientas que posibilitan la configuración sincrónica de distintas sendas de lectura.

Palabras clave: Lectura; crítica literaria; filología; manuscrito; medios electrónicos.
Abstract. The present paper provides a critical reflection about the importance of the contributions of the theoretical current known as New Philology, and more specifically to the ideas in the work In Praise of the of Variant (1999), as a founding work of this theoretical framework, and as a work of capital importance for the development of the philology discipline in recent years. Also, we want to focus the point of view on how these theoretical postulates and methodological aspects underlying as the conceptual essence for the development of new critical edition systems embedded in the digital paradigm. By means of that, explicit reference is made to digital systems for criticism of musical texts, which aim to incorporate variants presents in different testimonies, and the material particularities inherent to manuscripts through which they are transmitted, using tools that enable synchronous configuration of different reading paths.

Keywords: Electronic media; reading; philology; manuscripts; literary criticism.

\footnotetext{
${ }^{1}$ Para citar este artículo: Merchán Sánchez-Jara, Javier (2016). Del reconocimiento de la variante a la senda de lectura en el ámbito de la edición crítica digital. Alabe 13. [www.revistaalabe.com]

DOI: $10.15645 /$ Alabe2016.13.3
} 


\section{El Elogio de la Variantey la New Philology}

En su esencia más trascendental, la teoría postulada por Cerquiglini en su obra, (tan emblemática como polémica), In Praise of the Variant (1999) sostiene la visión del manuscrito medieval como una realidad multivalente, en la que la propia variación refleja el contexto líquido y en permanente relectura y renovación en el que se genera el texto medieval, como producto arquetípico de un paradigma basado en la trasmisión oral. Al amparo de esta tesis central se desgajan otras serie de consideraciones, que afectan a la manera en que estas propias variaciones han sido tratadas en el ámbito de la edición crítico-genética de estos materiales librarios. De esta manera, se aborda como a raíz del desarrollo de los métodos de impresión mecánica, se ha perseguido, durante largos periodos de tiempo y con afanes renovados, tanto la estabilización del texto, como la constitución y confirmación de la obra como entidad expresada dentro de una realidad única e inmutable.

El paradigma de la edición crítica moderna, surgido en el siglo XIX, ha perseguido como ideal platónico la reconstrucción, reelaboración y fijación de texto medieval (y de los textos en general) con el fin último de conseguir el texto arquetípico del que descienden los testimonios existentes, y bajo el marco de referencia que en la actualidad se tiene de conceptos como obra o autoría; o lo que es lo mismo, desde un paradigma moderno y descontextualizado con respecto al existente en la época de la que proceden, en el que se concibe la obra y el autor como entidades estancas, definidas y delimitadas; o lo que es lo mismo, el texto y la obra como expresión cultural de carácter totémico; y el autor como propietario y responsable único y último de la misma. En este contexto, la variación, o la cualidad de la variabilidad como rasgo caracterizador del texto medieval, se perciben de manera peyorativa, y en cierta forma, como amenaza a la inmutabilidad con la que desde el paradigma de la edición crítica se inviste al concepto de obra.

Cerquiglini refuerza esta serie de ideas a través de la contextualización de sus argumentaciones en una época como el siglo XIX, donde el concepto de autor y autoría adquiere la caracterización presente a día de hoy. El rechazo sistemático de los editores a las ocurrencias que expresan la variabilidad característica del manuscrito medieval, y su consideración como un elemento tóxico dentro del texto, como errores, está sin duda propiciado por una concepción monolítica tanto de la figura del autor como de las manifestaciones de su producción que permanece instalada de manera paradigmática en el lector post-medieval.

Las tesis postuladas para legitimar la presencia de la variación, son aproximadas no solo desde la perspectiva de que ésta constituye un elemento caracterizador del texto medieval y de su paradigma de producción, sino desde la perspectiva de que esta variación constituye, de igual manera, un elemento esencial en el repertorio que conforma la exigencia estética de este contexto histórico. Además, su puesta en valor incorpora y trasluce argumentos relacionados, por ejemplo, con la originalidad de cada obra gracias 
precisamente a la variación que contiene; es decir, la variación desde la perspectiva del elemento que aporta un carácter único a la obra y la convierte en ocurrencia diferenciada para ser disfrutada y puesta en valor.

De esta manera el manuscrito medieval, como objeto irrepetible que ejerce de pieza clave para la trasmisión y puesta en circulación del texto medieval, se convierte en una suerte de sistema en el que interactúan dimensiones como la autoría primigenia de la obra, y la intervención del copista; ésta, se produce en torno a procesos sistematizados y secuenciales de lectura, interpretación y reformulación interna de la información, y copia del mensaje reformulado. Es precisamente desde este proceso intelectual de reformulación del mensaje primigenio, donde surge la variación ${ }^{2}$, (que por otra parte inviste el rol del copista de un cierto carácter autorial), desde el cual podemos extraer información muy valiosa acerca de la prácticas lectoras de la época; mediante las distintas reformulaciones expresadas en los testimonios disponibles, o mediante los rasgos definitorios que caracterizan a los propios copistas a lo largo de su producción.

Por otro lado se requiere, de manera subsidiaria, de otro tipo de lectura que permita la consideración e interpretación, de manera concurrente con la lectura del texto, de los elementos semióticos que de manera incidental incorpora el manuscrito como objeto físico en relación con el conjunto de elementos paratextuales que incorpora, como en las particularidades de su manifestación material. Todas estas consideraciones hacen que sea necesario poner en liza, en el acercamiento al los manuscritos medievales, que generalmente portan y transmiten información muy valiosa en torno a las circunstancias relacionadas con el ecosistema cultural en el que se produce el testimonio (Graham D. Caie, 2008), dos estrategias de lectura; un centrada en la interpretación del contenido semántico (information-driven) , y otra (point-driven)(Douglas Vipond, 1984) que habilita al receptor a inferir propósitos, motivos o esquemas de reformulación en el autor, en este caso el copista.

Existen ejemplos de carácter ilustrativo en otros ámbitos que quizás resultan más inmediatos para comprender la magnitud de la significación de la variante, ya que se expresan, en cierta forma, de una manera más intuitiva. Uno de ellos es el ámbito musical, donde por un lado la interpretación del mensaje es una cuestión de relevancia crítica, y donde el contraste variación-repetición no solo constituye un elemento esencial a nivel estructural en la constitución del discurso musical, sino que de igual manera que en el ámbito literario medieval, este aparece como recurso estilístico o expresivo per se; o como en otras ocasiones, como el vehículo y el referente para el desarrollo de otros recursos expresivos como la ornamentación o la glosa (en el sentido de desarrollo y variación rítmico-melódica).

El desarrollo de la disciplina de la crítica textual (en menor medida la crítica especializada en textos musicales) se ha visto impregnada de continuos debates y confron-

\footnotetext{
${ }^{2}$ Nos referimos en este caso a todo el conjunto de variaciones que surgen al margen del error accidental.
} 
tación de perspectivas teóricas y / o metodológicas que han permitido, a través de los postulados de sus representantes más icónicos, la transición de un paradigma centrado en la concepción unitaria del binomio obra / autor, al reconocimiento más o menos generalizado de la variación como elemento consustancial a la obra manuscrita, generándose un continuo entre extremos que alberga un gran número de posturas intermedias; según Paris, por ejemplo, el escribano es el elemento en la cadena de transmisión de la obra a través del cual se germina y trasmite el error (la variación), por lo que una edición crítica debe de recorrer el camino inverso, desde los testimonios con los que se cuenta, en aras de identificarlo, solventarlo y restituir la obra a un estado lo más cercano a su concepción primigenia. Por otro lado, la postura de Bedier (una de las más comúnmente aceptadas) postula la edición individualizada de cada obra de acuerdo a su propia materialidad, y sin la referencia del resto de testimonios de la misma; así, cada testimonio produce una edición de la misma, única, individualizada y aislada del resto. Esta conceptualización representa, de modo sublimado, la idea de obra aislada, delimitada y cerrada que hemos comentado con anterioridad, por lo que en ambos casos, y como el propio Cerquiglini afirma en su afamada obra, se niega sistemáticamente la riqueza de la variación y la cualidad de la variabilidad; en el caso de Paris eliminándola y sustituyéndola por la hipotética verdad imaginada, y en el de Bedier al negar la estructura imaginaria que vincula a la obra matriz con sus testimonios. El texto de Cerquiglini, de carácter ciertamente provocador, tanto por el contenido, como por las formas (que en muchas ocasiones se alejan considerablemente de los estándares más flexibles de lo que debe considerarse un discurso de carácter academicista o científico) incorpora, al margen de las argumentaciones y consideraciones que hemos expuesto con anterioridad, las bases teóricas y conceptuales para constituirse como la obra fundacional (o la menos una de las más relevantes), del movimiento o escuela académica que conocemos como New Philology.

Si bien es cierto que la obra se constituye como una colección de ensayos interconectados de muy poca extensión, y no incorpora elementos destacables en el sentido de perfilarse como un manifiesto razonado y debidamente estructurado, su carácter sarcástico y la potencia de persuasión en el empleo del lenguaje por parte de Cerquiglini, la ha situado como una obra que a través de la polémica ha provocado que se abra el debate sobre las cuestiones que expone, y en consecuencia, que se haya podido generar un movimiento teórico en torno a esta. En cierta forma podemos resumir esta consideración, mediante la idea de que el movimiento se ha generado (independientemente de que existan bases epistemológicas para su constitución) más por la chispa de la polémica suscitada en torno a la obra de Cerquiglini, que por el contenido de la misma.En este sentido se echa quizás en falta en la obra una profundización mayor en torno a la interpretación de las implicaciones filológicas o literarias de la propia variación, y de la variabilidad. El autor se limita, en la mayoría de los casos, a defender con gran habilidad retórica su condición de defensor de una realidad cultural o histórica, y no a exponer ejemplos de cómo esta (la variación) afecta o que supone par el análisis filológico/ literario. Si bien es cierto que se describen abundantes ejemplos sobre como la variación se desarrolla en dos dimensiones 
(longitudinal, vertical), o sobre que facetas del discurso y sobre que elementos gramaticales o lingüísticos se reproduce; en ninguno de los casos se preocupa por argumentar ni a interpretar sus significación ni su aportación en el ámbito estricto del análisis filológico o literario.

En cualquier caso, la obra abrió el debate, y suscitó la necesidad de una renovación en las metodologías y las formas de aproximación a ciertas cuestiones y prácticas, asentadas y dadas por buenas durante largos periodos de tiempo.

Surge así el concepto de nueva filología, en primer lugar como respuesta a la percepción de que la filología medioeval había asido maltratada u olvidada por las nuevas metodologías de carácter cognitivo; y en segundo lugar, porque prácticas de estudio y análisis formuladas en el siglo XIX se seguían considerando, hasta hace pocos años, y de forma totalmente anacrónica, como actuales (Nichols, 1990).

Se desarrolla así un movimiento, que más que crear un nuevo paradigma (new philology), supone una renovación como vuelta al origen de la filología medieval centrada en el manuscrito, y en el paradigma de su producción, aceptando las características y las prácticas propias de este; entre ellas la variabilidad ${ }^{3}$.Este movimiento surge, de igual manera, (al margen del debate indicado por Cerquiglini), como fuerza opositora al concepto de filología heredado de la generación comandada por Auerbach. Ésta arranca de la normalización de la impresión mecánica en Europa, que conlleva el alejamiento sistemático de la representación de prácticas pasadas, en una cultura como la del manuscrito que incorpora la revisión, traducción, y rescritura de las obras en una experiencia más relacionada con la interpretación, que con la replicación. En este punto, el concepto de filología que hereda Auerbach determina su aspiración última, en la fijación del texto impreso de la forma más exacta y trasparente posible (Nichols, 1990), como vehículo para la actividad académica o investigadora; En este sentido, el objeto de estudio y el interés de la filología está representado en la idealización del texto, en detrimento de la materialidad del mismo.

Esta concepción, basada únicamente en el texto como manifestación (más próxima a la hipotética obra original), desprecia la aportación de toda una serie de artistas que de manera colaborativa intervienen directamente en la producción del manuscrito medieval; iluminadores, comentaristas, glosadores, escribas, poetas, etc. La aportación de cada uno de estos, además de constituir un sistema semiótico propio per se, interactúan con el resto de los que forman la obra de manera que esta no puede ser entendida y comprendida en su totalidad al margen de la propia materialidad del manuscrito.

Cualquiera de estas intervenciones en el pergamino supone, además, la aportación de la visión e interpretación del artista que las acomete, toda vez que estos no se limitan a una copia directa de la obra de la que se deriva, o a la que intentan reproducir. En este sentido el copista o escriba reinterpreta la obra, muchas veces como una especie

\footnotetext{
${ }^{3}$ Como el propio Cerquiglini argumenta en su obra, la escritura medieval no produce variantes; es varianza.
} 
de editor que juzga y aporta su visión de la misma, con intención de adaptarla o mejorarla, y muchas otras como el vehículo de trasmisión y constatación de cambios de percepción estética que operan en las distintas épocas (Nichols, 1990).

En definitiva, el paradigma de producción de estas obras incorpora como característica vertebradora, la multidimensionalidad, la variabilidad y la pluralidad; no como un accidente, si no como una cualidad cultivada de manera inherente.

Es en este punto donde la Nueva Filología adquiere sentido, como concienciación, aceptación e incorporación de esta característica tanto a los puntos de aproximación a la obra medieval, como a la metodología para su estudio o análisis.

\section{Del reconocimiento de la variabilidad a la senda de lectura}

Criticism is increasingly fond of whatever is unstable, multiple, and precarious, whatever temporarily goes beyond the enclosed immobility established by the machine. Note that this is happening at the very moment when the computer, the new technology, is producing floods of a writing that is mobile, various, and fluctuating. Does that mean that the written work is itself at stake? (Cerquiglini, 1999:13)

Con esta cita, inserta en el prólogo de la obra de Cerquiglini, (y que además se destaca en la cuarta de cubierta de la versión traducida en inglés), se pone de relieve de manera de manera profética, y 20 años antes de que los avances en las tecnologías de la información y la comunicación permitan su implementación, la esencia de los planteamientos más novedosos que se están desarrollando en relación a la edición crítica en la esfera digital. De hecho, y al margen de la reflexiones y la referencias explicitas a las potencialidades de la computadora y del texto digital, la obra en sí representa, analizándola desde una perspectiva con mayor grado de abstracción, no solo un elogio de la variante, sino un elogio de la hipertextualidad, y un elogio de la intertextualidad.

El propio Cerquiglini concluye su obra, (recordemos que la cita anterior se inserta en el prólogo de la obra), esta vez si de manera más explicita, relacionado estas potencialidades con la riqueza de las variantes que representa el manuscrito medieval. A través de un panegírico al medio digital, expone las posibilidades que incorpora en relación a la posibilidad de representación y de simulación de la riqueza inherente expresada en el manuscrito medieval: 
Elordenador, a través de su dialógica pantalla multidimensional, simula la movilidad sin fin y alegre de la escritura medieval, ya que restaura a su lector de la facultad asombrosa de la memoria $^{4}$ (Cerquiglini, 1999:79)

Pero quizás la reflexión más arriesgada, y que pone el foco en el asunto más esencial , es la que reza;

el ordenador es el medio que mejor representa el manuscrito medieval, en una forma que nunca alcanzan o no es alcanzable por la letra ${ }^{5}$ de la crítica edición (Cerquiglini, 1999:81)

Analizadas estas reflexiones, sin duda tan arriesgadas y especulativas en la época en la que se producen, como certeras e iluminadas vistas desde la perspectiva actual, es necesario observar con un poco más de detalle como se han ido concretando en los modelos desarrollados e implementados a día de hoy, tanto a nivel teórico como a nivel funcional. En este caso vamos a centrar el objeto de estudio en la implementación de estos sistemas para la edición crítica de manuscritos musicales, que salvando las distancias, y al margen de aspectos puntuales referidos al sistema semiótico empleado en la representación del discurso musical, guarda muchos paralelismos con los problemas y la casuística propia del ámbito de los manuscritos medievales.

Desde aproximadamente una década, la crítica textual ha experimentado una profunda reformación, no exenta de debate, gracias al desarrollo y la incorporación de las nuevas tecnología digitales a está practica. Los cambio más sustanciales acontecen, gracias a una serie de características esenciales que está presentes de manera recurrente en este ámbito; un lenguaje de marcado $(x m /)$ que permite separar la estructura lógica del contenido de su presentación; hecho que permite distintos modos de visualización, o la posibilidad de realizar operaciones y relaciones de tipo semántico, lógico, etc. Y por otro lado, la posibilidad de enriquecimiento o asociación de contenido paratextual (de carácter textual o multimedia) a cualquier parte o sección de la obra.

Estas dos consideraciones incorporan en el ámbito musical una serie de posibilidades que las distinguen de manera radical del paradigma de la partitura escrita; así por ejemplo, la representación del texto puede ser reproducido sonoramente; se pueden realizar transposiciones de tonalidad automáticas del mismo, o se pueden incorporar digitaciones, diccionarios y obras de referencia de escales arpegios, a cualquier parte 0 sección (Sánchez-Jara, 2014), etc.

\footnotetext{
${ }^{4}$ Traducción de la cita presente en la versión traducida al inglés.

${ }^{5}$ Entendemos que con el término letra se refiere a la expresión escrita, fija del paradigma impreso.
} 
Todas estas nuevas posibilidades se han desarrollado de manera específica y adaptada al ámbito de la edición, tanto en la esfera de la disciplina filológica como en la musicológica, con el desarrollo (aún en marcha) de sendos sistemas de codificación TEI (Text Encoding Initiative) y MEI (Music Encoding Initiative).

Pero, ¿cuáles es realmente la novedad más esencial que todas estas nuevas posibilidades brindan al mundo de la edición crítica musical?; la respuesta es tan sencilla, como compleja la implementación de las mismas; las posibilidades de la codificación digital permiten superar las limitaciones del formato físico; y ¿cuáles son esta limitaciones?; básicamente el formato impreso no permite representar, coordinar, ni relacionar todas las fuentes y los documentos en los que una edición critica está basada, y se limita a reproducirlas de manera fragmentada, esquemática, reducida, y no accesible, dentro el aparato crítico. Además, y como es lógico, deja fuera de igual manera cualquier referencia a materiales multimedia, como imagen, sonido, etc.

De esta manera, en la edición impresa es imposible el acceso completo a un testimonio o texto editado, y solo tenemos referencias parciales, sesgadas y descontextualizadas de su implicación en la edición, a través del aparato critico; y esto en el mejor de los casos, en el que el editor decida, en un punto de la obra editada, hacer referencia a ella. Nos encontramos, por lo tanto, ya desde un primer instante, con la disyuntiva sobre que aspectos y ocurrencias de la variación de la obra debemos referenciar, y cuales no; por lo que ello implica una primera negación a la cualidad del variabilidad en toda su dimensión.

Frente a esta inconveniencia del ámbito impreso se proponen, (MacGann, 1996) es el primero en hacerlo, sistemas integrados, o archivos hipermedia, que incorporan todos los testimonios a través de la representación virtual en forma de facsímiles digitales, (bien sea de naturaleza estática como PDF, o con contenido estructurado bajo lenguaje de marcado); un sistema lógico que los interconecta e interrelaciona; y herramientas de análisis para operar sobre ellos. Además, cada fuente puede incorporar sus propias anotaciones y /o referencias, es decir, su propio aparato crítico.

Por último, uno de los aspectos más esenciales respecto a las limitaciones de la edición crítica en el ámbito impreso, es que en la esfera de la edición tradicional, la dimensión de las fuentes queda toralmente independiente de la dimensión de la edición, toda vez que esta última recoge o condensa piezas o ocurrencias parciales de cada uno de ellas (ver ilustración 1). 


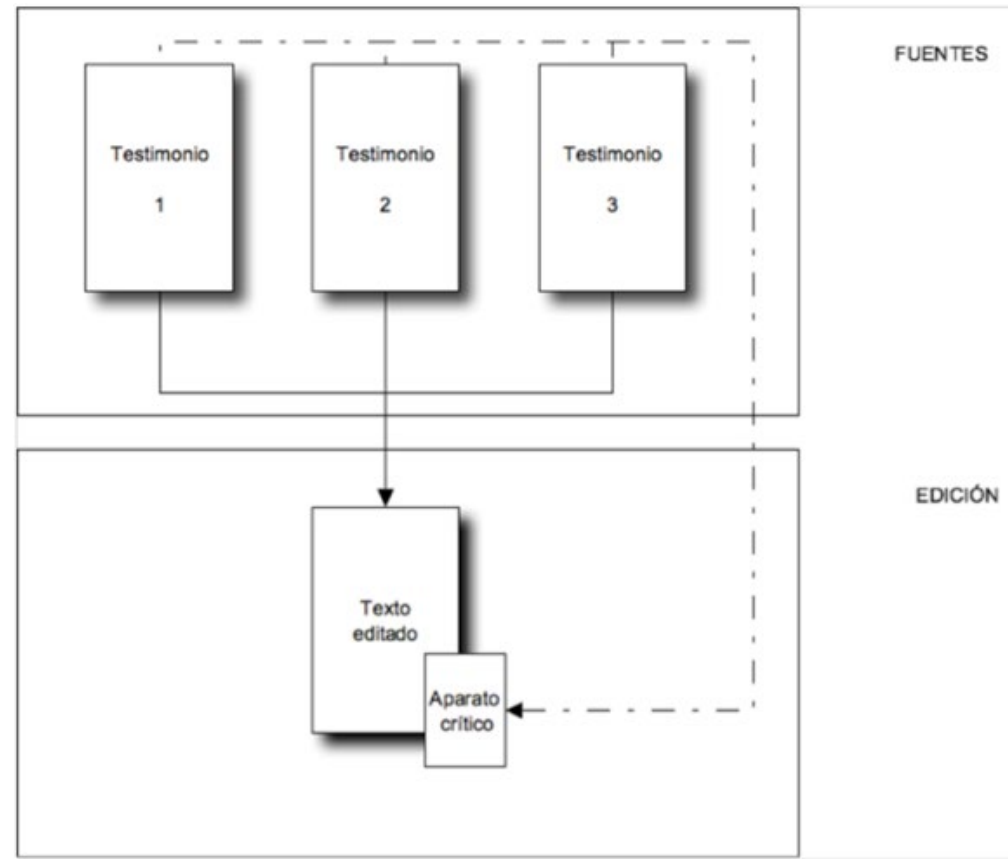

Ilustración 1. Ecosistema para la edición crítica en el ámbito impreso.

Fuente: elaboración propia

En el ámbito de la edición hipermedia, el ámbito de las fuentes y de la edición concurren en el mismo espacio y en el mismo plano, toda vez que la obra editada, de carácter virtual, se corresponde con un itinerario de lectura o senda de lectura (reading path), que puede ser múltiple, y editable de manera sincrónica, a través de los testimonios que están presentes, con su propio aparato crítico accesible en toda su dimensión. De esta manera se da cabida y relevancia a la variación expresada en los testimonios, toda vez que estos se encuentra presentes en todo momento, y pueden ser accedidos a través del sistema.( ver ilustración 2)

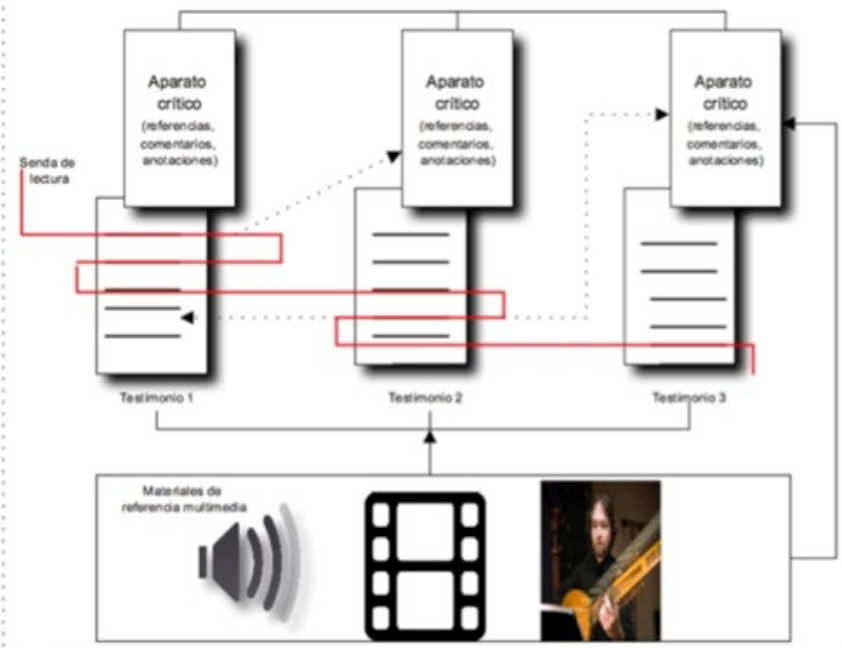

ESTRUCTURA CONJUNTA PARA Y EDICION

Ilustración 2. estructura conjunta y senda de lectura en edición hipermedia.

Fuente: elaboración propia. 
Este modelo o sistema hipermedia es especialmente adecuado para la edición crítica de textos musicales; por un lado permite, como hemos observado, contar de manera concurrente y relacionada con todos los testimonios de la obra, de manera que la riqueza de la variación en el sentido expresado por Cerquiglini, está presente y se pone de valor en todo momento; pero además, por que en el caso de la música, los textos musicales, al margen de constituirse únicamente como la representación de un mensaje musical tal como es concebido por el autor, representan marcos normativos que prescriben las directrices que indican como deben de ser ejecutados ( a nivel técnico y expresivo ) a través del medio instrumental o vocal. En este sentido el documento musical en forma de texto, (según distintos sistemas semióticos o de notación), incorpora una doble posibilidad de variabilidad; a nivel interpretativo, y a nivel de la propia semántica musical; es decir de la concepción de la propia obra musical.

Por otro lado, estos sistemas no pretenden de manera exclusiva la reconstrucción de la obra originaria o matriz, a través de una recreación hipotética o idealizada que se conforma a través de los testimonios, sino que posibilita el acceso puntual a cada uno de ellos según las distintas necesidades coyunturales que puedan presentarse; pero además, se posibilita la configuración de versiones estables que representen la edición más próxima a la obra ideal en el sentido filogenético, según distintas consideraciones y criterios, y a modo de la edición tradicional, a través de las denominadas rutas o sendas de lectura, o mediante la configuración de un texto principal.

En el ámbito musical este aspecto además incorpora otras consideraciones de gran interés; en este sentido la edición crítica musical a través de estos sistemas digitales permite adoptar diversas manifestaciones creadas ad hoc según, por ejemplo, las distintas corrientes estéticas que ha postulado lecturas fuertes ${ }^{6}$ en cada época, ediciones adaptadas o conformadas en torno a los percepción de distintas escuelas interpretativas o técnicas, etc.

Finalmente debemos apuntar que todas estas posibilidades y consideraciones están en cierta manera motivadas y son posibles gracias a la presencia de la variante; en este sentido no solo representan, como postulaba Cerquiglini, una realidad constatable e inherente a un paradigma de producción de obras manuscritas que hay que considerar como expresión propia de la época; en el ámbito musical, estas variantes incorporan, de igual manera, un carácter teleológico muy marcado y muy influyente en cuestiones como la compresión global de la obra; la observación de las distintas formas de aproximación a la interpretación, o la ejecución a nivel técnico de ciertos pasajes; o como ha afectado a la propia obra, su recepción en las distintas épocas o paradigmas estéticos por los que ha transitado.

\footnotetext{
${ }^{6}$ Entendido en el sentido que le otorga Harold Bloom en el contexto del canon literario.
} 


\section{Bibliografía}

- Cerquiglini, B. (1999). In Praise of the Variant: A Critical History of Philology. (1st ed.). Baltimore: Johns Hopkins University Press.

- Douglas Vipond, R. A. H. (1984). Point-driven understanding: Pragmatic and cognitive dimensions of literary reading. Poetics, 13(3), 261-277. http://doi.org/10.1016/0304$422 X(84) 90005-6$

- Graham D. Caie, D. R. (2008). Medieval texts in context (1st ed.). London: Routledge. McGann, J. (1996). The Rationale of Hyper Text. Text, 9, 11-32.

- Nichols, S. G. (1990). Introduction: Philology in a Manuscript Culture. Speculum, 65(1), 1. http://doi.org/10.2307/2864468

- Sánchez-Jara, J. M. (2014, October). Digital schola: music readers as learning/teaching tools. In Proceedings of the Second International Conference on Technological Ecosystems for Enhancing Multiculturality (pp. 547-553). ACM. 